\title{
Membrane and membrane-associated proteins involved in the aggressive phenotype displayed by highly invasive cancer cells
}

\author{
Paul Dowling, Naomi Walsh and Martin Clynes \\ The National Institute for Cellular Biotechnology, Dublin City University, Dublin, Ireland
}

\begin{abstract}
Invasion, the penetration of tumour cells into adjacent tissues, is a fundamental characteristic of malignant carcinomas and a first step in the metastatic process. The molecular mechanisms involved in tumour cell invasion are complex, but over the last couple of decades the knowledge base has grown quite considerably and many proteins with important roles in invasion have been identified and characterised. Benign tumours typically are encapsulated, which inhibits their ability to behave in a malignant manner, meaning these tumours do not grow in a location-limited less aggressive manner, do not invade surrounding tissues and do not metastasise. The ability of malignant tumours to invade and metastasise is the major cause of death for cancer patients. A greater insight into the molecular basis of cancer invasion and metastasis will lead to the development of novel therapies and specific panels of biomarkers for use in the treatment and diagnosis/monitoring in many types of metastatic cancer.
\end{abstract}

Received: January 31, 2008

Revised: April 18, 2008

Accepted: April 24, 2008

\section{Keywords:}

Integrins / Invasion / Markers / Membrane / Receptors

\section{Introduction}

The abilities of cells to invade and metastasise are critical features of cancer. Metastasis is defined by the ability of tumour cells at the primary site to invade local tissue, cross

Correspondence: Dr. Paul Dowling, National Institute for Cellular Biotechnology, Dublin City University, Glasnevin, Dublin 9, Ireland

E-mail: paul.dowling@dcu.ie

Fax: +353-1-7005484

Abbreviations: aODN, antisense oligonucleotide; BAC, benzyldimethyl-n-hexadecylammonium chloride; CAM, cell adhesion molecule; ECM, extracellular matrix; EGF, epidermal growth factor; EGFR, epidermal growth factor receptor; EMT, epithelial to mesenchymal transition; FALI, fluorophore-assisted light inactivation; HGF, hepatocyte growth factor; IHC, immuno-histochemistry; MMP, matrix metalloproteinase; NCAM, neural-CAM; PM, plasma membrane; SILAC, stable isotope labelling with amino acids in cell culture; TMD, transmembrane domain; uPA, urokinase-type plasminogen activator; uPAR, urokinase plasminogen activator receptor basement membranes/tissue barriers and to re-establish at distant secondary locations. To initiate separation of a malignant cell from the tumour mass, cancer cells must first break signalling contact with neighbouring cells, degrade and penetrate the basement membrane and then invade the interstitial stroma in order to reach blood/lymph vessels [1]. Intravasation requires penetration of the blood/lymph systems. The tumour cells must then exit the lymph system or blood stream at a new site (extravasation) and proliferate in the secondary organ [2].

The three step model for tumour invasion (degradation, adhesion and migration of cancer cells) has proved to be the basis for much research in the field of tumour invasion and metastasis [3]. Degradation of the basement membrane and interstitial connective tissue requires the production of extracellular proteases that are secreted in high concentrations from the invading tumour front.

During the metastatic cascade adhesion molecules seem to be missing or are compromised. For example, epithelial (E)-cadherin is frequently partly or entirely downregulated in cancer cells, facilitating detachment from each other and from the extracellular matrix (ECM). To migrate, the cell 
body must modify its shape and elasticity to interact with the surrounding tissue structures. Cell migration through tissues results from a continuous cycle of steps, including the formation of lamellipodia, filopodia, pseudopodia or invadopodia at certain distinct time points during the migration process.

This review will focus on membrane and membraneassociated protein involvement in many of the interdependent steps in tumour cell invasion, how this leads to metastatic growth in distant organs and the impact of particular protein as drug targets and as markers of disease state.

\section{In vitro model of invasion}

\subsection{General}

Typically, during tumour progression and malignant transformation, epithelial cells acquire mesenchymal properties because of the epithelial to mesenchymal transition (EMT). EMT is a typical feature of aggressive, invasive and metastatic cancer cells. In vitro, the morphology of highly invasive cancer cells can reflect the characteristics of EMT, such as loss of cell adhesion (E-cadherin) and increased cell motility. Cells undergoing EMT display a scattered spindle shaped morphology, whereas noninvasive cells remain in tight compact epithelial colonies (Figs. 1A and B).

Various in vitro models have been developed to investigate the molecular mechanisms associated with invasion and to explore the effects of certain pharmacological agents on this process. As with most cell culture models, the in vitro invasion assay may not be a perfect representation of the in vivo phenotype, but over the last number of decades it has proved to be a valuable source of information and has undoubtedly increased our overall understanding of the complex interdependent processes involved in cancer cell invasion. Two main areas in proteomics, namely expression and function-based platforms, have been extensively exam-
A.

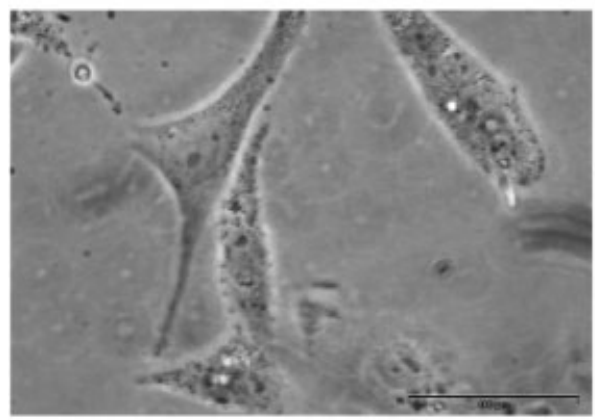

c.

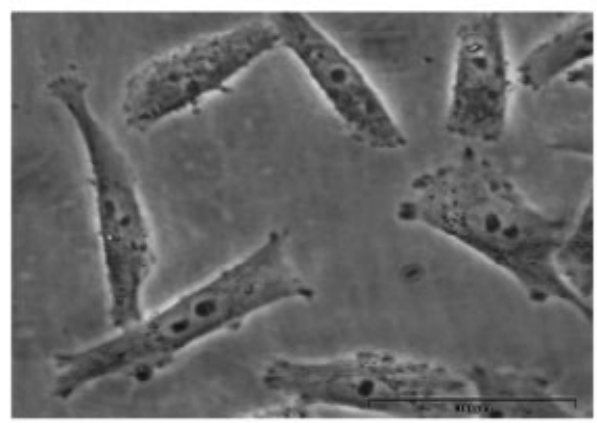

E.

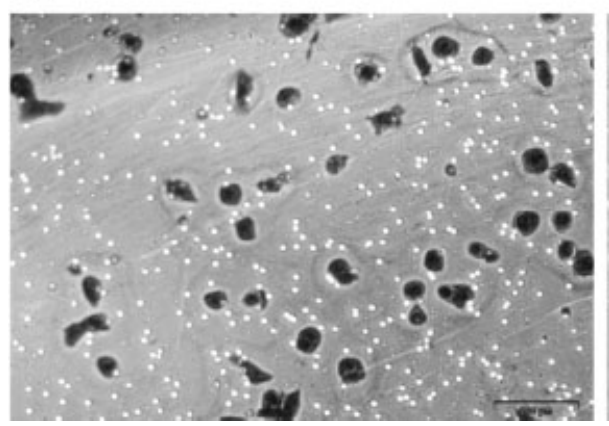

B.

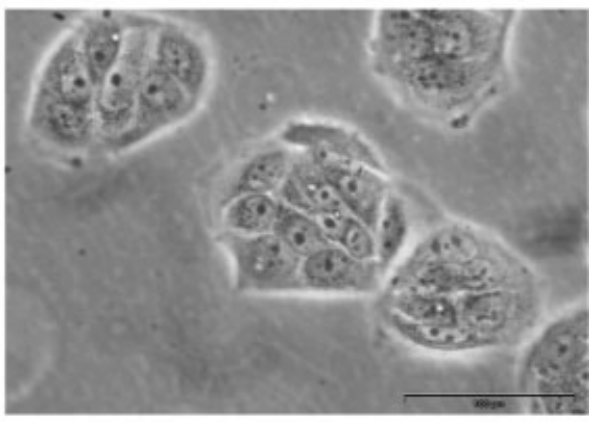

D.

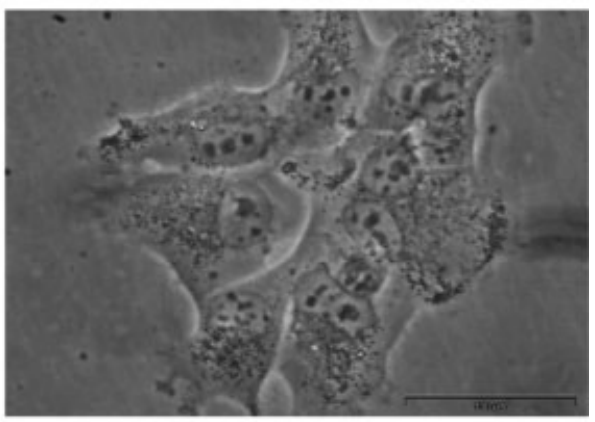

F.

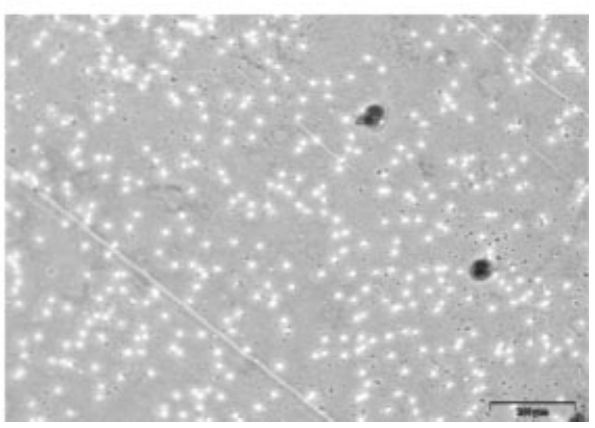

Figure 1. (A) Morphology of a highly invasive breast cancer cell line, MDA-MB-231 with elongated and spindle-like phenotype. (B) Noninvasive breast cancer cell line, MCF-7 with epithelial tight colonies. (C) and (D) Highly invasive and non/low invasive clonal populations of the pancreatic cancer cell line, MiaPaCa-2 displaying characteristic morphology. Magnification, $400 \times$. Scale bar $100 \mu \mathrm{m}$. (E) Cell invasion assay representing the highly invasive pancreatic clonal cell line invading through ECM coated Boyden chamber, stained with crystal violet. (F) Cell invasion assay representing the low/noninvasive pancreatic clonal cell line invading through ECM coated Boyden chamber. Magnification, $200 \times$. Scale bar $200 \mu \mathrm{m}$. 
ined using cell culture models of invasion. Expression proteomics has allowed for the identification of proteins found to have changed abundance levels in invasive compared to noninvasive phenotypes. Functional proteomics approaches have facilitated researchers gaining more concentrated information on specific proteins through the use of siRNA knockdown and cDNA overexpression systems, modern imaging analysis and protein-protein interaction studies.

\subsection{Invasion assay}

The Boyden chamber chemoinvasion assay, developed first by Albini et al. [4] is still widely used to study the invasive potential of cell culture models in vitro. In general, cells are placed in the upper compartment and are allowed to migrate through the pores of the membrane into the lower compartment, in which chemotactic agents are present. Using different matrices to mimic the basement membrane, the invasion chamber serves as a model to investigate the tumour cells ability to penetrate into and through the ECM and towards a chemoattractant. Pro- or anti-invasion factors can be added to the upper chamber containing the tumour cells to investigate their specific effects. Cells are given a fixed period of time to adhere to and penetrate the ECM with noninvading cells located on the upper surface of the filter and invading cells on the lower chamber. Invading tumour cells are generally fixed, stained and counted thereafter or used to establish an invasive subpopulation of tumour cells (Figs. 1C and D). Many different aspects of the assay can be altered including the size of the pores in the membrane, the coating of the lower chamber including the use of collagen I, fibronectin or gelatin which serve as chemoattractants and also the cell density used initially.

The selection of cancer cell lines with chemotherapeutic agents can alter the invasive potential of cells, resulting in a superinvasive phenotype, where cells not only invade through Matrigel and migrate through membrane pores, but also subsequently survive in suspension and ultimately attach to and grow on the bottom of the well beneath the insert. In our laboratories, Glynn et al. [5] demonstrated that selection of the human cell line MDA-MB-435S-F with doxorubicin and paclitaxel resulted in a more aggressively invasive phenotype, termed superinvasion.

\section{Extracellular matrix (ECM) degradation}

Degradation of the ECM is an essential step in tumour cell invasion and metastasis. The ECM contains many components including proteoglycans, collagens, fibronectins, elastins and laminins. Integrins are cell surface proteins that bind cells to ECM structures, such as fibronectin and laminin, activating pathways that lead to the degradation of the ECM by specific enzymes such as the matrix metalloproteinases (MMPs) and the urokinase-type plasminogen activator (uPA) system in invasive tumour cells.

\subsection{Integrins}

As the main link between a cell and the ECM, integrins have an essential role in the invasion process. Integrins are a large family of heterodimeric proteins which transduce a variety of signals from fibronectin and other ECM molecules. They are composed of a larger alpha $(\alpha)$ and smaller beta $(\beta)$ subunits. Both contain a large extracellular domain, a membranespanning region, and a relatively small cytoplasmic domain. The extracellular parts of the two subunits associate noncovalently to form a functional receptor. Integrins are formed from $18 \alpha$ and $8 \beta$ subunits, which dimerise to yield at least 24 different integrin heterodimers, each with distinct ligand binding and signalling properties. Ligand occupancy is critical for activation of integrin signalling involving many different pathways and the fibronectin-integrin interactions are important in tumour cell migration, invasion and metastasis.

The role for specific integrins and their associated ligands in intracellular signalling pathways has been described in a number of tumour types, and often multiple integrins are involved within a single tumour type. For example, during attachment to lymph nodes, melanoma cells utilise $\alpha_{\mathrm{v}} \beta_{3}$-vitronectin binding interactions, whereas breast cancer cells utilise integrin $\alpha_{3} \beta_{1}$-fibronectin binding [6]. Integrin $\alpha_{2} \beta_{1}$ was originally characterised as a melanoma progression antigen and measurement of this receptor might be used as predictive markers when assessing disease progression [7].

Modern proteomics labelling technologies have been employed by several groups to identify integrins and other proteins involved in specific invasive phenotypes being investigated. Chen et al. [8] used ICAT (isotope-coded affinity tag) and MS/MS to perform quantitative proteomic profiling of pancreatic cancer tissues and normal pancreas to identify proteins associated with invasion and metastasis. Their results indicated many proteins with elevated abundance levels in the pancreatic cancer proteome compared to the control, including integrin $\beta_{1}$, a result that was confirmed by Western blotting and immuno-histochemistry (IHC). Using iTRAQ reagents, followed by 2-D LC-MS/MS, Keshamouni et al. [9] identified a total of 51 differentially expressed proteins during TGF-beta induced-EMT in the human lung cancer cell line A549. Proteins found upregulated included integrin $\beta 1$ and cofilin, suggesting the acquisition of an invasive phenotype.

Integrin-mediated degradation of the ECM and associated tissue is an important first step of invasion. The role of the integrins is not limited to their function as a mechanical bond in cell-matrix contact sites, but, after binding extracellular ligands, integrins are also capable of sending signals into the cell. The basement membrane is a thin sheet composed of a mixture of ECM proteins, including laminins, collagens and proteoglycans produced in part by the epithelial cells themselves and in part by underlying connective tissue cells (specifically, fibroblasts). Interference in the regular functioning of the basement membrane is a key histo- 
logical marker of a tumour's transition to an invasive carcinoma. Degradation of the basement membrane requires the expression of specific proteases that target unique members of the ECM. This targeting by certain proteases in tightly regulated, with a continually changing balance existing between active proteases and their inhibitors, preventing excess matrix degradation.

Amongst the proteases activated by up-stream stimulation of integrin receptors are a family of proteins named the MMPs. They are active on a relatively broad range of targets including collagen, plasminogen, elastin, fibronectin, laminin and the urokinase plasminogen activator receptor (uPAR). MMPs are secreted as inactive zymogens (proMMPs) that require proteolytic activation, much of which occurs at the cell surface and involves many membrane or membrane-associated proteins. For example, cell surface activation of an MMP can occur when gelatinase A (MMP-2) is brought into contact with a membrane-associated MMP, MT-1 MMP [10, 11]. MMP-2 and MMP-9 (gelatinase B) have their greatest enzymatic activities against type IV collagen, which is the main constituent of the basement membrane $[12,13]$.

Integrins can regulate the expression of the MMPs genes and they have also been shown to influence the secretion of the granule-stored MMPs and the activation of the latent protease at the cell surface. One of the best characterised of these pathways is the mitogen activated protein kinase (MAPK) cascade. This serial phosphorylation of substrate proteins terminates in activation of transcription factors which regulate expression of target genes. Many of these genes are critical for ECM degradation or cell migration.

A complex series of interactions, starting with the initial stimulation of the integrin receptor and subsequent activation of intracellular pathways are responsible for maintaining the precise degradation on the ECM. In the course of tumour cell invasion and migration, interactions with integrins and the ECM are constantly been formed and broken and depending on the type of ligand occupying the receptor site, a different series on intracellular cascades are set in motion.

\subsection{UPAR and galectins}

An important factor implicated in tumour cell predisposition for invasion is the malignancy-related upregulation of UPAR. UPAR is a cysteine rich glycoprotein (about 55$60 \mathrm{kDa}$ ) attached to the plasma membrane (PM) via its carboxy-terminal to a glycosyl phosphatidyl inositol (GPI) anchor. uPAR can bind to available pro-uPA and, upon activation, uPA can bring about degradation of ECM leading to invasion/metastasis by stimulating tyrosine kinase and subsequent downstream signalling pathways. Margheri et al. recently examined the effects of inhibiting uPAR signalling by inhibition of UPAR expression with antisense oligonucleotides (aODNs) in PC3 human prostate cancer cells and evaluated aODN effect in a mouse model of prostate cancer bone metastasis. There results demonstrated that PC3 cells adhesion was unaffected, while proliferation and invasion of Matrigel were impaired. Mice injected with PC3 cells and treated with aODN to uPAR revealed an $80 \%$ reduction in bone metastasis as compared to the control groups [14].

Galectins have important functions in cell-cell interactions, cell adhesion to ECM, the organisation of ECM and tissue remodelling. Although galectins lack a transmembrane domain (TMD), these proteins are known to function at the cell surface. Galectins seem to play a role in the expression of integrins on the cell surface, promoting interactions with fibronectin, laminin, vitronectin, collagen and other cell membrane proteins. Altered expression levels of galectin-1 and galectin-3 are often associated with the invasive potential of cancer cells. Tsuboi et al. [15] recently showed that the reduction of galectin-3 expression is associated with the invasion and metastasis of colorectal cancer using immunohistochemical assessment at the tumour surface and the invasive front. A possible involvement of galectin-3 expression in tumour invasion, metastasis and proliferation in patients with colorectal cancer is suggested. Employing IHC and reverse transcriptionquantitative PCR assay Chiang et al. [16] suggest that galectin-1 upregulation at the tumour invasion front might be a predictor of early metastasis in oral carcinogenesis. Previous work on galectin-3 in our laboratory showed that galectin-3 cDNA transfection into the human nonsmall cell lung carcinoma cell line, DLKP, resulted in enhanced adhesion to ECM components, cell motility and in vitro invasiveness [17]. Another study in our laboratory demonstrated increased levels of galectin-3 in superinvasive cells (Fig. 2) from isolated membrane fractions using 2-D DIGE and MS [18]. These superinvasive cells also displayed reduced attachment to ECM proteins coupled with increased motility.

\section{Adhesion proteins}

Interactions either among cells (cell-cell) or between cells and substratum components (cell-matrix) are mediated by adhesion molecules. Cancer cells have been shown to express most of the adhesion receptors found on the surface of normal cells. Changes in the abundance levels of various adhesion molecules at specific time points have been proposed to be necessary in order for the complex process of invasion to progress [19]. More recently, adhesion molecules have been considered as potential biomarkers in the diagnosis, prognosis and treatment in many cases of human cancer. Cell surface adhesion molecules play vital roles in numerous cellular processes, including cancer cell invasion. There are five major families of cell adhesion molecules (CAMs) including Ig superfamily CAMs, integrins, cadherins, selectins and CD44. 

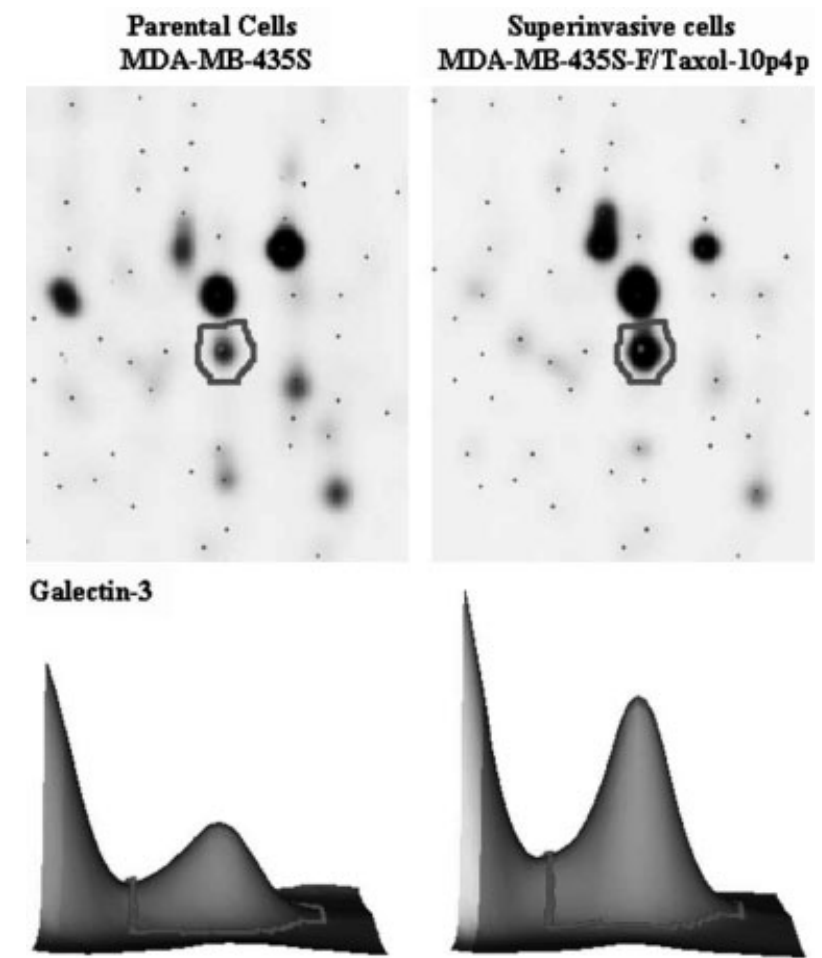

Figure 2. 2-D DIGE analysis of MDA-MB-435S-F and MDA-MB$435 \mathrm{~S}-\mathrm{F} / \mathrm{Tax}$ l10p4pSI membrane proteins. Shown here is the abundance levels for Galectin-3, a protein involved in cancer cell invasion. Galectin-3 was found to be approximately two-fold increased in the superinvasive cells compared to the parental cells. Images were generated using the BVA module of DeCyder software. Galectin-3 was identified using LC-MS yielding four matched peptides (FNENNR; VIVCNTK; IALDFOR; LDNNWGR) resulting in a $17.4 \%$ sequence coverage.

\subsection{Cadherins}

Cadherins are cell surface membrane glycoproteins capable of mediating cell-cell adhesion in a calcium-dependent fashion. The three most common cadherins are neural (N)cadherin, placental $(\mathrm{P})$-cadherin and E-cadherin. Many in vitro and in vivo studies primarily focusing on E-cadherin have demonstrating that loss of expression and function is closely related to induction of invasiveness and metastatic potential [20, 21].

E-cadherins mediate homotypic adhesions in epithelial tissue, bonding epithelial cells together and relaying signals between the cells. In several epithelial tumours, including adenocarcinomas of the colon and breast, there is a downregulation of E-cadherin expression. The down-regulation reduces the ability of cells to adhere to each other and facilitates their detachment from the primary tumour and their advance into the surrounding tissues. It has been proposed that the loss of E-cadherin-mediated cell-cell adhesion is a prerequisite for tumour cell invasion and metastasis formation [22].
Jiang et al. combined 2-DE separation and MS identification to analyse a pair of highly and poorly metastatic sublines (termed PLA801D and PLA801C, respectively). Ectopically expressed interleukin 18 (IL-18) promoted cell motility in vitro and downregulated E-cadherin expression of PLA801C transfectants, while IL-18 antisense remarkably decreased cell invasion potency in vitro and notably increased E-cadherin expression of PLA801D transfectants [23].

E-cadherins are linked to the cytoskeleton by the catenins, proteins that lie under the PM. The normal function of E-cadherin is dependent on its linkage to catenins. In some tumours, E-cadherin is normal, but its expression is reduced because of mutations in the gene for a catenin [24].

The loss of E-cadherin function during tumour progression can be caused by various genetic or epigenetic mechanisms. In some cancers, including bladder and gastric, the Ecadherin gene is mutated, leading to the expression of a nonfunctional protein [25]. In the majority of cancers, Ecadherin expression is downregulated at the transcriptional level, by proteins including Snail and Slug that bind to E2 boxes in the promoter of the E-cadherin gene [26].

In several human cancer types, including melanoma, prostate and breast cancer, loss of E-cadherin function is accompanied by de novo expression of mesenchymal cadherins, such as N-cadherin and cadherin-11. The switch from Ecadherin to $\mathrm{N}$-cadherin expression might provoke the tumour cell to move into different surroundings and invading the underlying stroma. E-cadherin is expressed by epithelial cells whereas mesenchymal cadherins are found in stromal cells such as fibroblasts [27].

\subsection{Igs and CD44}

The Ig superfamily CAMs are calcium-independent transmembrane glycoproteins. Members of the Ig superfamily include the intercellular adhesion molecules (ICAMs), vascular-CAM (VCAM-1), platelet-endothelial-CAM (PECAM-1) and neural-CAM (NCAM). Each Ig superfamily CAM has an extracellular domain, which contains several Ig-like intrachain disulphide-bonded loops with conserved cysteine residues, a TMD and an intracellular domain that interacts with the cytoskeleton. Typically, they bind integrins or other Ig superfamily CAMs.

Some CAMs of the Ig superfamily are often found to have altered levels during the growth and progression of certain tumours. These alterations in abundance levels of certain Ig's on the cell surface may facilitate migration of tumour cells from the primary lesion through their adhesive activities, resulting in invasion and disseminating metastasis [28]. Three major isoforms of NCAM are generated by alternative splicing, which differ in their membrane association and their intracellular portions: NCAM $180(180 \mathrm{kDa})$ and NCAM $140(140 \mathrm{kDa})$ are transmembrane proteins with cytoplasmic tails of different lengths, whereas the $120 \mathrm{kDa}$ isoform NCAM 120 is anchored to the membrane by means of glycosylphosphatidyl-inositol 
(GPI). In various tumour types, N-CAM expression shifts from the adult $120 \mathrm{kDa}$ isoform to the embryonic 140 and $180 \mathrm{kDa}$ isoforms [29], increasing the invasive potential of the cancer cells. Perl et al. [30] demonstrated that using the Rip1Tag2 transgenic mouse model, the abrogation of $\mathrm{N}$ CAM expression in pancreatic $\beta$-cell tumours resulted in a dramatic increase in the incidence of metastases, predominantly to local lymph nodes. The precise mechanisms by which N-CAM impacts on the invasive potential of many tumour types remains incomplete, but the interaction with the fibroblast growth factor receptor (FGFR) is crucial leading to the activation of a complex series of intracellular events [31, 32].

The CD44 protein is a type I transmembrane glycoprotein receptor involved in cell-cell interactions, cell adhesion and migration. The principal ligand of CD44 is hyaluronic acid, an integral component of the ECM. Other CD44 ligands include osteopontin, serglycin, collagens, fibronectin and laminin. Stimulation of CD44 by one of its various ligands results in intracellular signalling involving Rho/Ras and activated tyrosine kinase pathways as well as the proteolytic generation of a fragment of CD44 that seems important as a signalling molecule. Lopez et al. reported that invasion of CD44-positive tumour cells was inhibited in hyaluronancontaining matrices, whereas blocking CD44-hyaluronan association increased invasion. Collectively, this data shows that during breast cancer progression, hyaluronan-CD44 dynamics occurring through epithelial-stromal interactions are protective against metastasis [33].

\section{Membrane and membrane-associated proteins involved in cell migration}

Cell migration is a critical step in tumour invasion and metastasis. Cell migration involves multiple processes that are regulated by various molecules including many membrane and membrane-associated proteins. The actin cytoskeleton and its regulatory proteins are crucial for cell migration in invasive tumour cells. Actin cytoskeletal reorganisation is essential for tumour cell migration, adhesion and invasion.

\subsection{Actin remodelling}

Cells use two kinds of protrusions, filopodia and lamellipodia, to migrate forward propelled by actin polymerisation at the leading edge. Filopodia and lamellipodia differ in respect to their actin organisation and the signalling pathways that are activated.

Degradation of the ECM by cancer or stromal cells involves specialised actin-rich adhesions, called podosomes and invadopodia. Both structures are dependent on actin organisation and dynamics, similar to lamellipodia and filopodia. Podosomes and invadopodia contain a variety of proteins involved with actin filaments, actin binding proteins, adhesion proteins, matrix proteinases and signalling proteins that regulate the actin cytoskeleton and membrane remodelling.

Extensive actin polymerisation at the leading edge requires the activity of cofilin, a member of the $\mathrm{ADF} /$ cofilin family that associates with cytoskeletal proteins when activated. Cofilin is essential for the regulation of actin polymerisation and depolymerisation during tumour cell motility and invasion. Upon receptor stimulation, cofilin nucleates actin polymerisation by severing actin filaments to generate free barbed ends and also increases the rate of actin depolymerisation. Yamaguchi et al. proposed a model for invadopodium formation where cofilin may be required for optimising Arp2/3 complex-mediated dendritic nucleation to cause elongation of the invadopodia and its stabilisation. From data collected in our research laboratories, a significant increase in the abundance of cofilin and several cytoskeletal structural/regulatory proteins was found to have an important role in mechanisms associated with superinvasion and other invasive model systems [18, 34-37].

Beckner et al. described a novel two-step method for harvesting pseudopodia (invadopodia) to provide material for proteomic analysis. Differences in the protein profiles of pseudopodia and whole cells were found using DIGE and immunoblotting. Met and actin along with other proteins associated with invasion and metastasis were increased in pseudopodial lysates on immunoblots when compared to whole cells and according to the author, represent therapeutic targets to consider for suppression of tumour invasion [38].

\subsection{Receptors stimulation and cytoskeletal reorganisation}

Cell migration and invasion are triggered by a number of chemoattractants. Upon binding to cell surface receptors, these chemoattractants stimulate intracellular signalling pathways that regulate reorganisation of the actin cytoskeleton. In many different cancer cell types the prognosis of a patient is inversely correlated with the overexpression and/or amplification of the epidermal growth factor receptor (EGFR) family [39]. Accumulating evidence suggests that EGFR ligands and the EGFR signalling pathway are critical for the invasiveness and metastasis of cancer cells (Fig. 3). The EGFR (EGFR; ErbB-1; HER1 in humans) is the cell-surface receptor for members of the epidermal growth factor (EGF)-family of extracellular protein ligands. The EGFR is a member of the ErbB family of receptors, a subfamily of four closely related receptor tyrosine kinases: EGFR (ErbB-1), HER2/c-neu (ErbB-2), Her 3 (ErbB-3) and Her 4 (ErbB-4).

Yamaguchi et al. [40] demonstrated that EGFR signalling is necessary for invadopodium formation and may be an initial key step of cancer cell invasion and metastasis induced by activation of EGFR signalling. Stimulation of the EGFR, recruitment and activation of Nck1 and Cdc42 leading to the activated Neural Wiskott-Aldrich Syndrome Protein 


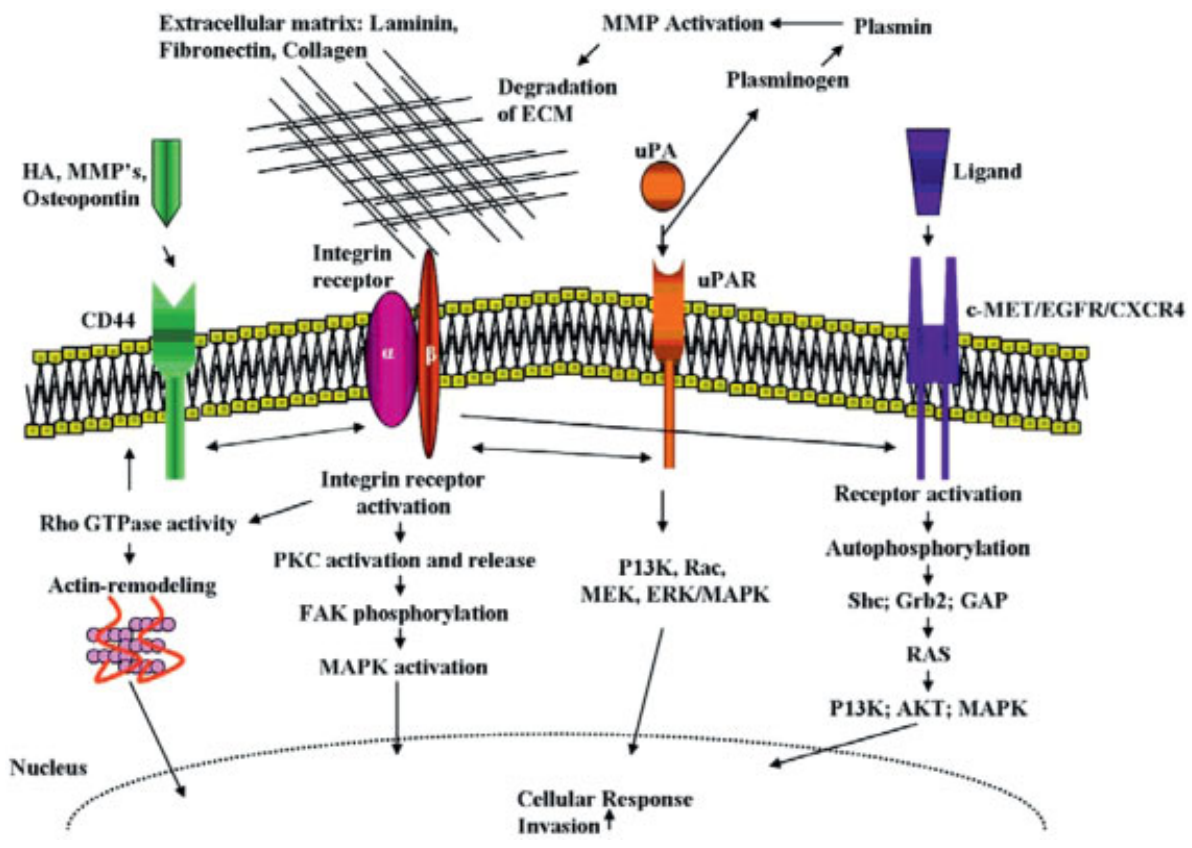

Figure 3. Invasive tumour cell. This diagram represents some of the membrane and membrane-associated proteins involved in the invasive phenotype of many tumour cells. The activation of many membrane proteins including CD44 and the integrin receptors co-activates the expression of many other membrane proteins leading to an increase in the invasive potential of tumour cells. Also shown here is a selection of intracellular and extracellular events involved in invasion.
(N-WASP) induces actin polymerisation through Arp2/3 complex that drives invadopodium initiation [41]. In our laboratory, it has been reported that EGFR was elevated in cells found to display a superinvasive phenotype when compared to the parental cells [5].

Interactions between carcinoma cells and macrophages promote tumour invasion and metastasis. This promotion of invasion is mediated by a paracrine loop between the two cell types involving EGF and CSF-1 (colony-stimulating factor-1) that enhances chemotactic cell migration of the two cell types. Macrophages along with other cell types secrete EGF that stimulates the formation of invadpodia through a series of intracellular pathways. CSF-1 is a central element in the paracrine loop, and is produced by carcinoma cells thereby acting to recruit macrophages to the tumour cells through the stimulation of the macrophage colony-stimulating factor receptors and subsequently promoting invasion. Yamaguchi et al. [42] have also shown that macrophage podosomes can degrade the ECM after localising to the site of the tumour, assisting in the invasive process.

The hepatocyte growth factor (HGF) receptor, also called c-Met, is activated by HGF, a paracrine factor secreted by stromal and mesenchymal cells. c-Met is overexpressed or modified in a variety of carcinomas including lung, pancreas and breast [43]. In patients with invasive breast cancer, Met or HGF expression is an independent predictor of poor prognosis [44]. HGF and Met have been implicated in the modulation of the actin cytoskeleton and in the secretion of MMPs that degrade and remodel the ECM in certain carcinomas including human synovial sarcoma [45, 46]. Davies et al. [47] demonstrated that by targeting the HGF/scatter factor (SF) receptor c-met using a hammerhead ribozyme trans- gene, they could reduce in vitro invasion and migration in prostate cancer cells. In our laboratory, it has been reported that a $50 \mathrm{kDa}$ truncated form of c-Met was elevated in MDAMB-435S-F/Adr-10p10pSI, a superinvasive population of cells when compared to the parental cells [5].

CXCR4 also called fusin, is a chemokine receptor in the GPCR gene family, and is expressed by cells in the immune system and the CNS. In response to binding its ligand SDF-1 (stromal cell-derived factor-1/CXC chemokine ligand-12), CXCR4 triggers the migration/recruitment of immune cells and activates downstream pathways such as ras and PI3 kinase. Breast cancer cells expressing CXCR4 undergo actin cytoskeleton remodelling upon SDF-1 stimulation, resulting in the formation of actin-filled protrusions [48].

\section{Membrane proteins as diagnostic/ prognostic markers and therapeutic targets}

Membrane and membrane-associated proteins involved in the invasive potential of tumour cells and subsequent metastasise to secondary sites are a major source of prognostic indicators in a variety of cancers. For example, CAMs, such as E-cadherin, integrins and CD44 appear to have some prognostic significance. Many membrane and membraneassociated proteins involved in cancer invasion are attractive targets for pharmaceutical intervention. Integrins have become attractive targets for drug development, and several effective integrin antagonists are now under clinical evaluation. Limited agents targeted at tumour or endothelial cell adhesion has entered clinical trials, but several are under 
development. New therapeutics feature the use of peptidomimetics and $\mathrm{mAb}$ targeted against many membrane and membrane-associated proteins.

\subsection{Diagnostic/prognostic markers}

Loss of E-cadherin expression has been shown to correlate with invasion and metastasis in many types of carcinoma [49]. Madhavan et al. [50] reported that the expression of Ecadherin and P-cadherin showed significant down-regulation in node-positive tumours compared to node-negative tumours when examined by IHC in 51 cases of breast cancer that included 29 node-negative and 22 node-positive cases.

Integrins are actively involved in the invasive potential of tumour cell, with different isoforms associated with particular cancers and the aggressiveness of the malignancy. Grossman et al. examined frozen sections of bladder tumours, urine specimens, and bladder wash specimens from 59 patients, comparing the level of integrin $\alpha_{6} \beta_{4}$ with patient outcomes. Patients with the invasive form of cancer who strongly expressed $\alpha_{6} \beta_{4}$ had a significantly shorter survival time than patients who expressed $\alpha_{6} \beta_{4}$ weakly or not at all [51]. Levels of integrins in biofluids such as serum, is a significant development allowing for rapid screening and investigation into the status of the tumour with respect to the invasive and metastatic potential. Ke et al. have recently reported that integrin $\beta_{1}$ levels in serum were highly expressed in patients with gastric carcinoma before operation or with unresectable tumours compared to a control group. The expression levels decreased substantially in the postoperative patients subjected to radical resection of the tumours, indicating that the high level expressions of integrin $\beta_{1}$ might be the important factor for predicting the prognosis of these patients [52].

CD44 proteins are released in soluble form (solCD44) by the action of MMP (MT1-MMP). The soluble 70-kDa fragment is detectable in blood where its levels are correlated with increased invasive and metastatic potential in some tumours [53, 54].

Galectin-3, a member of the $\beta$-galactoside-binding animal lectins, has been implicated in tumour invasion and metastasis. Serum levels of galectin-3 have been reported to predict progression and the invasive and metastatic potential of many cancers. Iurisci et al. assessed the circulating levels of galectin-3 in sera from cancer patients as well as from healthy controls. Low serum levels of galectin-3 were detected in healthy individuals (median, $62 \mathrm{ng} / \mathrm{mL}$ ). Compared with healthy individuals, galectin-3 serum levels in patients with breast, gastrointestinal, lung or ovarian cancer, melanoma and non-Hodgkin's lymphoma were significantly elevated. Moreover, galectin-3 concentrations in sera from patients with metastatic disease were higher than in sera from patients with localised tumours. Maximum serum concentrations of galectin-3 (median, $320 \mathrm{ng} / \mathrm{mL}$ ) were found in patients with metastatic gastrointestinal carcinoma [55].
Reports on EGFR and associated receptors have established a link between serum levels of these receptors and the invasive and metastatic potential of certain cancers. The extracellular binding region of these two receptors for EGFR and HER2 are proteolytically released from the cell surface upon receptor activation and can be detected in patients' serum. In a study performed by Asgeirsson et al., both sEGFR and sHER2 levels changed significantly between the times of primary and metastatic diagnosis. Serum EGFR levels were found to be significantly higher in healthy individuals than in primary breast cancer patients [56]. Quaranta et al. discovered that the EGFR was elevated in 47 out of 65 brain cancer patients, with mean serum values of $84 \mathrm{ng} / \mathrm{mL}$, compared with that of healthy controls which was $43.6 \mathrm{ng} /$ $\mathrm{mL}$ and concluded that measurement in serum of the EGFR extracellular domain may be potentially useful as a biological marker of gliomas for the prediction of prognosis and followup after treatment [57].

\subsection{Therapeutic targets}

The $\alpha_{\mathrm{v}} \beta_{3}$ integrin is an adhesion receptor implicated in tumour cell invasion and osteoclast-mediated bone resorption. Zhao et al. data show that tumour $\alpha_{\mathrm{v}} \beta_{3}$ integrin stands as a therapeutic target for the prevention of skeletal metastases. The nonpeptide antagonist of $\alpha_{\mathrm{v}} \beta_{3}$ (PSK1404) showed a meaningful antitumour effects in experimental breast and ovarian cancer bone metastasis [58]. Vitaxin, an antihuman $\alpha_{\mathrm{v}} \beta_{3}$ antibody is currently in clinical trials as a treatment for metastatic melanoma and results indicate that this drug may be instrumental in inhibiting the advancement of lifethreatening cancers [59].

$\mathrm{N}$-cadherin which is associated with increased tumour invasiveness is a potential novel therapeutic target. The epithelial-mesenchymal transition characterised by the loss of E-cadherin and the de novo expression of N-cadherin adhesion molecules, highlights $\mathrm{N}$-cadherin as a major target for pharmaceutical intervention. A peptidic N-cadherin antagonist (ADH-1) has been developed and has entered clinical testing. Shintani et al. [60] recently demonstrated that the Ncadherin antagonist, ADH-1 has significant antitumour activity against $\mathrm{N}$-cadherin-expressing cells using in vitro assays and in an orthotopic mouse model for pancreatic cancer, raising the possibility that $\mathrm{N}$-cadherin antagonists have therapeutic potential for the treatment of pancreatic cancer in humans.

Abnormalities in EGFR functions are associated with all key features of cancer development and growth, including invasion and metastatic potential. Aberrant EGFR signalling can be initiated by several events, such as altered ligand production, receptor mutations or deletions and persistent activation. High levels of EGFR expression are a common feature of the malignant phenotype in many solid human tumours. Numerous EGFR inhibitors are under development, for example gefitinib and erlotinib that inhibit the receptor's intracellular tyrosine kinase activity as well as 
cetuximab and panitumumab that block extracellular ligand binding. EGFR inhibitors have been shown to effectively decrease the invasive and metastatic potential of many types of aggressive tumours [61].

A wide variety of human cancers exhibit constitutively dysregulated c-Met activity, either through overexpression of the c-Met kinase, activating mutations in c-Met, or increased autocrine or paracrine secretion of the c-Met ligand, HGF/ $\mathrm{SF}$. Increased expression of the receptor tyrosine kinase cMet has been shown to correlate with enhanced motility and invasion. Despite the promise of c-Met as a cancer target, the discovery of a selective c-Met inhibitor has proved to be a difficult challenge. Koon et al. [62] recently reported that using a specific adenosine triphosphate-competitive smallmolecule inhibitor, SU11274, activated c-Met was decreased in normal and ovarian carcinoma cell lines resulting in a significant decreased in cell motility and invasion compared to untreated cells.

Cancer invasion and metastasis have also been linked to cellular communication via the CXCR4 activated pathways and receptor inhibitors could potentially prove very important as a treatment strategy. Kim et al. [63] have recently demonstrated that inhibition of the CXCR4/CXCL12 pathway decreases metastatic disease in two murine tumour models using CTCE-9908, a small peptide CXCR4 antagonist.

\section{Modern strategies for the expression and functional proteomic analysis of membrane and membrane-associated proteins}

Gel-based strategies for studying membrane proteins at a proteomic scale often involve the separation of intact membrane proteins using 2-DE, proving especially suitable for membrane proteins that contain only one TMD. 2-DE is a powerful electrophoretic method for the separation of complex protein samples that applies IEF in the first dimension and SDS-PAGE in the second dimension. The addition of DIGE technology that labels protein samples with fluorescent Cye dyes prior to electrophoresis, enables accurate analysis of differences in protein abundance between samples and has added greatly to the overall usability of gel-based systems for proteins separation.

However this technique does not resolve membrane proteins that contain more than one TMD very well due to their high hydrophobicity resulting in low solubility and a high tendency for aggregation. Recently alternative methods for performing conventional 2-DE on membrane and membrane-associated proteins have been described and successfully demonstrated. Zahedi et al. [64] showed the effectiveness of 2-D benzyldimethyl- $n$-hexadecylammonium chloride (BAC)/SDS-PAGE (2-DB) using the cationic detergent BAC in the first and the anionic detergent SDS in the second dimension as a powerful tool for the separation and analysis of membrane proteins. The 2-DB enabled the identification of extremely hydrophobic proteins including cytochrome $c$ oxidase subunit I from Saccharomyces cerevisiae with a total of 12 known TMD's. Membrane proteins identified using 2-DB demonstrated significantly higher hydrophobicity, as measured by increased GRAVY indices [65]. As an alternative to BAC, Helling et al. [66] used 2-D cetyl trimethyl ammonium bromide (CTAB)/SDS-PAGE with fluorescence dye saturation labelling (satDIGE) to analyse small quantities of membrane proteins from mouse T-cells. The results demonstrated how valuable and reproducible this technique is for the separation/quantification of scarce membrane proteins.

Separation of membrane proteins by 1-D SDS-PAGE and identification by in-gel trypsin digestion and MS analysis has proved useful for analysing small complexes of hydrophobic proteins. However for more complex samples, 1-D SDSPAGE alone is insufficient for adequate separation as demonstrated by Moebius et al. [67] who reported finding ten or more proteins per band in a typical 1-DE separation. The 1-DE can be combined with many chromatographic techniques such as RP separation of proteins, and has proved to be very successful in the analysis of complex membrane proteins. Cao et al. [68] analysed isolated PM proteins using SDS-PAGE and microcapillary RP LC-MS/MS to identify 428 proteins, including 204 integral membrane or membraneassociated proteins.

New advances in quantitative proteomic analysis including stable isotope labelling with amino acids in cell culture (SILAC), ICAT and iTRAQ as well as multidimensional protein identification technology (MudPIT) have increased the capability of proteomic methods to study the expression levels of membrane proteins. Together with MS-based proteomics strategies, such technologies have allowed for the analysis and quantitation of many membrane and membrane-associated proteins previously found to be difficult to perform. SILAC has proven to be very successful for the identification of membrane proteins in various cell culture models. Quantification of membrane and membrane-bound proteins in normal and malignant breast cancer cells was performed by Liang et al. [69] using SILAC. Proteins identified in this study included $\mathrm{G}$ proteins, $\mathrm{G}$ binding proteins, farnesyltransferases, ephrins, integrins, porins, $\alpha$-catenin and EGFR precursor demonstrating how powerful SILAC is for the analysis of membrane and membrane-associated proteins. Given the large amount of cell culture models available for work on cancer invasion and metastasis, SILAC will undoubtedly prove to be popular for the identification of membrane proteins associated with these phenotypes. Using ICAT, Ramus et al. [70] showed that labelling of highly hydrophobic proteins with cleavable ICAT is possible and may even be improved in strong denaturing buffers containing both SDS at a concentration higher than $0.5 \% \mathrm{w} / \mathrm{v}$ and urea.

Cell culture models are especially useful when studying the roles of specific membrane and membrane-associated proteins in invasion and metastasis. Using siRNA knock- 
down and/or cDNA overexpression coupled with a cell culture-based assay (invasion/motility/adhesion), the particular function of proteins can be assessed to a greater extent. Recently new functional proteomics screens using fluorophore-assisted light inactivation (FALI) for acute protein knockdown directed by specific binders have been developed. FALI is a high-throughput method of damaging proteins in situ by using fluorescein-labelled binders and $490 \mathrm{~nm}$ diffuse light [71]. Eustace et al. used both RNAmediated interference (RNAi) and FALI screens to identify the molecular chaperone hsp90 as an important extracellular mediator of invasion. Inhibition of extracellular hsp90 decreases both MMP2 activity and invasiveness demonstrating the effectiveness of both functional screens [72].

Membrane and membrane-associated proteins form part of complexes that work together to perform specific processes within a cell. Identification of all the members of particular complexes is important to the overall understanding of how individual proteins participate and interact with each other. Routine cloning techniques, tagging systems including His/GST/Flag tags and associated purification columns allow for the isolation of specific protein complexes and their identification through MS [73]. Cell culture models of cancer invasion and metastasis also afford the opportunity to image protein localisation and protein-protein interactions in live cells. New fluorescent labelling technologies coupled with advanced light microscope techniques make visualising and monitoring processes in living cells accessible. Fusion membrane or membrane-associated proteins containing GFP sequences may be recombinantly expressed and subsequently monitored in the intact cell with the use of fluorescence resonance energy transfer (FRET) for example [74].

\section{Conclusions and perspectives}

Membrane and membrane-associated proteins play a vital role in numerous cellular functions. We have summarised here the involvement of many membrane proteins in tumour cell invasion. The majority of membrane proteins involved in tumour progression are generally of low abundance, difficult to solubilise and can contain numerous PTMs making analysis more difficult than soluble proteins. The rapid growth in proteomics technologies, including separation techniques coupled with MS has also coincided with advances in sample preparation and protein isolation, especially for membrane proteins making analysis now more assessable [75-77].

Many of these proteins are found to be active in many of the interdependent stages of tumour progression and have a variety of complex functions as part of the malignancy. Membrane proteins are attractive as therapeutic targets and many new treatment regimes target specific molecules involved in tumour cell invasion successfully. Many mem- brane proteins are now actively employed as markers of disease state, providing valuable information that allows for greater control of therapeutic choice.

The authors have declared no conflict of interest.

\section{References}

[1] Ray, J. M., Stetler-Stevenson, W. G., The role of matrix metalloproteases and their inhibitors in tumour invasion, metastasis and angiogenesis. Eur. Respir. J. 1994, 7, 20622072.

[2] Deryugina, E. I., Bourdon, M. A., Tenascin mediates human glioma cell migration and modulates cell migration on fibronectin. J. Cell Sci. 1996, 109, 643-652.

[3] Stetler-Stevenson, W. G., Aznavoorian, S., Liotta, L. A., Tumor cell interactions with the extracellular matrix during invasion and metastasis. Annu. Rev. Cell Biol. 2003, 9, 541573.

[4] Albini, A., Iwamoto, Y., Kleinman, H. K., Martin, G. R. et al., A rapid in vitro assay for quantitating the invasive potential of tumor cells. Cancer Res. 1987, 47, 3239-3245.

[5] Glynn, S. A., Gammell, P., Heenan, M., O'Connor, R. et al., A new superinvasive in vitro phenotype induced by selection of human breast carcinoma cells with the chemotherapeutic drugs paclitaxel and doxorubicin. Br. J. Cancer 2004, 15, 1800-1807.

[6] Felding-Habermann, B., Integrin adhesion receptors in tumor metastasis. Clin. Exp. Metastasis 2003, 20, 203-213.

[7] Han, J., Jenq, W., Kefalides, N. A., Integrin alpha2beta1 recognizes laminin-2 and induces $\mathrm{C}$-erb $\mathrm{B} 2$ tyrosine phosphorylation in metastatic human melanoma cells. Connect. Tissue Res. 1999, 40, 283-293.

[8] Chen, R., Yi, E. C., Donohoe, D., Pan, S. et al., Pancreatic cancer proteome: The proteins that underlie invasion, metastasis, and immunologic escape. Gastroenterology 2005, 129, 1187-1197.

[9] Keshamouni, V. G., Michailidis, G., Grasso, C. S., Anthwal, S., Differential protein expression profiling by iTRAQ-2DLCMS/MS of lung cancer cells undergoing epithelial-mesenchymal transition reveals a migratory/invasive phenotype. J. Proteome Res. 2006, 5, 1143-1154.

[10] Sato, H., Takino, T., Okada, Y., Cao, J. et al., A matrix metalloproteinase expressed on the surface of invasive tumour cells. Nature 1994, 370, 61-65.

[11] Strongin, A. Y., Collier, I., Bannikov, G., Marmer, B. L. et al., Mechanism of cell surface activation of 72-kDa type IV collagenase. Isolation of the activated form of the membrane metalloprotease. J. Biol. Chem. 1995, 270, 5331-5338.

[12] Collier, I. E., Wilhelm, S. M., Eisen, A. Z., Marmer, B. L. et al., $\mathrm{H}$-ras oncogene-transformed human bronchial epithelial cells (TBE-1) secrete a single metalloprotease capable of degrading basement membrane collagen. J. Biol. Chem. 1988, 263, 6579-6587.

[13] Cortes-Reynosa, P., Robledo, T., Macias-Silva, M., Wu, S. V., Salazar, E. P., Src kinase regulates metalloproteinase-9 secretion induced by type IV collagen in MCF-7 human breast cancer cells. Matrix Biol. 2008, 27, 220-231. 
[14] Margheri, F., D’Alessio, S., Serratí, S., Pucci, M. et al., Effects of blocking urokinase receptor signaling by antisense oligonucleotides in a mouse model of experimental prostate cancer bone metastases. Gene Ther. 2005, 12, 702-714.

[15] Tsuboi, K., Shimura, T., Masuda, N., Ide, M. et al., Galectin-3 expression in colorectal cancer: Relation to invasion and metastasis. Anticancer Res. 2007, 27, 2289-2296.

[16] Chiang, W. F., Liu, S. Y., Fang, L. Y., Lin, C. N. et al., Overexpression of galectin-1 at the tumor invasion front is associated with poor prognosis in early-stage oral squamous cell carcinoma. Oral Oncol. 2008, 44, 325-334.

[17] O’Driscoll, L., Linehan, R., Liang, Y. H., Joyce, H. et al., Galectin-3 expression alters adhesion, motility and invasion in a lung cell line (DLKP), in vitro. Anticancer Res. 2002, 22, 3117-3125.

[18] Dowling, P., Meleady, P., Dowd, A., Henry, M. et al., Proteomic analysis of isolated membrane fractions from superinvasive cancer cells. Biochim. Biophys. Acta 2007, 1774, 93101.

[19] Charalabopoulos, K., Papalimneou, V., Charalabopoulos, A., Adhesion molecules in lung cancer. Exp. Oncol. 2003, 25, 16-21.

[20] Yuecheng, Y., Hongmei, L., Xiaoyan, X., Clinical evaluation of E-cadherin expression and its regulation mechanism in epithelial ovarian cancer. Clin. Exp. Metastasis 2006, 23, 6574.

[21] Yi, D., Kyoon, J., Kyu, Y., Yeob, S. et al., E-cadherin expression in early gastric carcinoma and correlation with lymph node metastasis. J. Surg. Oncol. 2007, 96, 429-435.

[22] Cavallaro, U., Christofori, G., Cell adhesion and signalling by cadherins and Ig-CAMs in cancer. Nat. Rev. Cancer 2004, 4, 118-132.

[23] Jiang, D., Ying, W., Lu, Y., Wan, J. et al., Identification of metastasis-associated proteins by proteomic analysis and functional exploration of interleukin-18 in metastasis. Proteomics 2003, 3, 724-737.

[24] Vermeulen, S. J., Nollet, F., Teugels, E., Vennekens, K. M. et al., The alphaE-catenin gene (CTNNA1) acts as an invasionsuppressor gene in human colon cancer cells. Oncogene 1999, 18, 905-915.

[25] Strathdee, G., Epigenetic versus genetic alterations in the inactivation of E-cadherin. Semin. Cancer Biol. 2002, 12, 373-379.

[26] Batlle, E., Sancho, E., Francí, C., Domínguez, D. et al., The transcription factor snail is a repressor of E-cadherin gene expression in epithelial tumour cells. Nat. Cell Biol. 2002, 2, 84-89.

[27] Tomita, K., van Bokhoven, A., van Leenders, G. J., Ruijter, E. T. et al., Cadherin switching in human prostate cancer progression. Cancer Res. 2000, 60, 3650-3654.

[28] Skubitz, A. P., Adhesion molecules. Cancer Treat. Res. 2002, 107, 305-329.

[29] Kaiser, U., Auerbach, B., Oldenburg, M., The neural cell adhesion molecule NCAM in multiple myeloma. Leuk. Lymphoma 1996, 20, 389-395.

[30] Perl, A. K., Dahl, U., Wilgenbus, P., Cremer, H. et al., Reduced expression of neural cell adhesion molecule induces metastatic dissemination of pancreatic beta tumor cells. Nat. Med. 1999, 5, 286-291.
[31] Cavallaro, U., Niedermeyer, J., Fuxa, M., Christofori, G., NCAM modulates tumour-cell adhesion to matrix by inducing FGF-receptor signalling. Nat. Cell Biol. 2001, 3, 650-657.

[32] Petersen, L. G., Størling, J., Heding, P., Li, S. et al., IL-1betainduced pro-apoptotic signalling is facilitated by NCAM/FGF receptor signalling and inhibited by the C3d ligand in the INS-1E rat beta cell line. Diabetologia 2006, 49, 1864-1875.

[33] Lopez, J. I., Camenisch, T. D., Stevens, M. V., Sands, B. J. et al., CD44 attenuates metastatic invasion during breast cancer progression. Cancer Res. 2005, 65, 6755-6763.

[34] Carlier, M. F., Ressad, F., Pantaloni, D., Control of actin dynamics in cell motility. Role of ADF/cofilin. J. Biol. Chem. 1999, 274, 33827-33830.

[36] Loisel, T. P., Boujemaa, R., Pantaloni, D., Carlier, M. F., Reconstitution of actin-based motility of Listeria and Shigella using pure proteins. Nature 1999, 401, 613-616.

[37] Chan, A. Y., Bailly, M., Zebda, N., Segall, J. E., Condeelis, J. S., Role of cofilin in epidermal growth factor-stimulated actin polymerization and lamellipod protrusion. J. Cell. Biol. $2000,148,531-542$.

[38] Yamaguchi, H., Wyckoff, J., Condeelis, J., Cell migration in tumors. Curr. Opin. Cell Biol. 2005, 17, 559-564.

[39] Beckner, M. E., Chen, X., An, J., Day, B. W., Pollack, I. F., Proteomic characterization of harvested pseudopodia with differential gel electrophoresis and specific antibodies. Lab. Invest. 2005, 85, 316-327.

[40] Nicholson, R. I., Gee, J. M., Harper, M. E., EGFR and cancer prognosis. Eur. J. Cancer 2001, 37, S9-S15.

[41] Yamaguchi, H., Lorenz, M., Kempiak, S., Sarmiento, C. et al., Molecular mechanisms of invadopodium formation: The role of the N-WASP-Arp2/3 complex pathway and cofilin. $J$. Cell Biol. 2005, 168, 441-452.

[42] Lorenz, M., Yamaguchi, H., Wang, Y., Singer, R. H., Condeelis, J., Imaging sites of N-WASP activity in lamellipodia and invadopodia of carcinoma cells. Curr. Biol. 2004, 14, 697-703.

[43] Yamaguchi, H., Pixley, F., Condeelis, J., Invadopodia and podosomes in tumor invasion. Eur. J. Cell Biol. 2006, 85, 213-218.

[44] Ma, P. C., Tretiakova, M. S., Nallasura, V., Jagadeeswaran, R. et al., Downstream signalling and specific inhibition of cMET/HGF pathway in small cell lung cancer: Implications for tumour invasion. Br. J. Cancer 2007, 97, 368-377.

[45] Birchmeier, C., Birchmeier, W., Gherardi, E., Vande Woude, G. F., Met, metastasis, motility and more. Nat. Rev. Mol. Cell Biol. 2003, 4, 915-925.

[46] Ma, P. C., Maulik, G., Christensen, J., Salgia, R., c-Met: Structure, functions and potential for therapeutic inhibition. Cancer Metastasis Rev. 2003, 22, 309-325.

[47] Watanabe, T., Tsuda, M., Makino, Y., Ichihara, S. et al., Adaptor molecule Crk is required for sustained phosphorylation of Grb2-associated binder 1 and hepatocyte growth factor-induced cell motility of human synovial sarcoma cell lines. Mol. Cancer Res. 2006, 4, 499-510.

[48] Davies, G., Watkins, G., Mason, M. D., Jiang, W. G., Targeting the HGF/SF receptor c-met using a hammerhead ribozyme transgene reduces in vitro invasion and migration in prostate cancer cells. Prostate 2004, 60, 317-324.

[49] Holland, J. D., Kochetkova, M., Akekawatchai, C., Dottore, M. et al., Differential functional activation of chemokine recep- 
tor CXCR4 is mediated by $\mathrm{G}$ proteins in breast cancer cells. Cancer Res. 2006, 66, 4117-4124.

[50] Tada, H., Hatoko, M., Muramatsu, T., Shirai, T., Expression of E-cadherin in skin carcinomas. J. Dermatol. 1996, 23, 104110.

[51] Madhavan, M., Srinivas, P., Abraham, E., Ahmed, I. et al., Cadherins as predictive markers of nodal metastasis in breast cancer. Mod. Pathol. 2001, 14, 423-427.

[52] Grossman, H. B., Lee, C., Bromberg, J., Liebert, M., Expression of the alpha6beta4 integrin provides prognostic information in bladder cancer. Oncol. Rep. 2000, 7, 13-16.

[53] Ke, J. J., Shao, Q. S., Ling, Z. Q., Expression of E-selectin, integrin beta 1 and immunoglobulin superfamily member in human gastric carcinoma cells and its clinicopathologic significance. World J. Gastroenterol. 2006, 12, 3609-3611.

[54] Kajita, M., Itoh, Y., Chiba, T., Mori, H. et al., Membrane-type 1 matrix metalloproteinase cleaves CD44 and promotes cell migration. J. Cell Biol. 2001, 153, 893-904.

[55] Yamane, N., Tsujitani, S., Makino, M., Maeta, M., Kaibara, N., Soluble CD44 variant 6 as a prognostic indicator in patients with colorectal cancer. Oncology 1999, 56, 232-238.

[56] Iurisci, I., Tinari, N., Natoli, C., Angelucci, D. et al., Concentrations of galectin-3 in the sera of normal controls and cancer patients. Clin. Cancer Res. 2000, 6, 1389-1393.

[57] Asgeirsson, K. S., Agrawal, A., Allen, C., Hitch, A. et al., Serum epidermal growth factor receptor and HER2 expression in primary and metastatic breast cancer patients. Breast Cancer Res. 2007, 9, R75.

[58] Quaranta, M., Divella, R., Daniele, A., Di Tardo, S. et al., Epidermal growth factor receptor serum levels and prognostic value in malignant gliomas. Tumori 2007, 93, 275-280.

[59] Zhao, Y., Bachelier, R., Treilleux, I., Pujuguet, P. et al., Tumor alphavbeta3 integrin is a therapeutic target for breast cancer bone metastases. Cancer Res. 2007, 67, 5821-5830.

[60] Tucker, G. C., Integrins: Molecular targets in cancer therapy. Curr. Oncol. Rep. 2006, 8, 96-103.

[61] Shintani, Y., Fukumoto, Y., Chaika, N., Grandgenett, P. M. et al., ADH-1 suppresses N-cadherin-dependent pancreatic cancer progression. Int. J. Cancer 2008, 122, 71-77.

[62] Normanno, N., Gullick, W. J., Epidermal growth factor receptor tyrosine kinase inhibitors and bone metastases: Different mechanisms of action for a novel therapeutic application? Endocr. Relat. Cancer 2006, 13, 3-6.

[63] Koon, E. C., Ma, P. C., Salgia, R., Welch, W. R. et al., Effect of a c-Met-specific, ATP-competitive small-molecule inhibitor SU11274 on human ovarian carcinoma cell growth, motility, and invasion. Int. J. Gynecol. Cancer 2007 (Epub ahead of print), doi: 10.1111/j.1525-1438.2007.01135.x.

[64] Kim, S. Y., Lee, C. H., Midura, B. V., Yeung, C. et al., Inhibition of the CXCR4/CXCL12 chemokine pathway reduces the development of murine pulmonary metastases. Clin. Exp. Metastasis 2008, 25, 201-211.
[65] Zahedi, R. P., Moebius, J., Sickmann, A., Two-dimensional BAC/SDS-PAGE for membrane proteomics. Subcell. Biochem. 2007, 43, 13-20.

[66] Coughenour, H. D., Spaulding, R. S., Thompson, C. M., The synaptic vesicle proteome: A comparative study in membrane protein identification. Proteomics 2004, 4, 3141-3155.

[67] Helling, S., Schmitt, E., Joppich, C., Schulenborg, T. et al., 2D differential membrane proteome analysis of scarce protein samples. Proteomics 2006, 6, 4506-4513.

[68] Moebius, J., Zahedi, R. P., Lewandrowski, U., Berger, C. et al., The human platelet membrane proteome reveals several new potential membrane proteins. Mol. Cell. Proteomics 2005, 4, 1754.

[69] Cao, R., Li, X., Liu, Z., Peng, X. et al., Integration of a twophase partition method into proteomics research on rat liver plasma membrane proteins. J. Proteome Res. 2006, 5, 634642.

[70] Liang, X., Zhao, J., Hajivandi, M., Wu, R. et al., Quantification of membrane and membrane-bound proteins in normal and malignant breast cancer cells isolated from the same patient with primary breast carcinoma. J. Proteome Res. 2006, 5, 2632-2641.

[71] Ramus, C., Gonzalez de Peredo, A., Dahout, C., Gallagher, M. et al., An optimized strategy for ICAT quantification of membrane proteins. Mol. Cell. Proteomics 2006, 5, 68-78.

[72] Beck, S., Sakurai, T., Eustace, B. K., Beste, G. et al., Fluorophore-assisted light inactivation: A high-throughput tool for direct target validation of proteins. Proteomics 2002, 2, 247-255.

[73] Eustace, B. K., Sakurai, T., Stewart, J. K., Yimlamai, D. et al., Functional proteomic screens reveal an essential extracellular role for hsp90 alpha in cancer cell invasiveness. Nat. Cell Biol. 2004, 6, 507-514.

[74] Cross, M., Nguyen, T., Bogdanoska, V., Reynolds, E. et al., A proteomics strategy for the enrichment of receptor-associated complexes. Proteomics 2005, 5, 4754-4763.

[75] Bene, L., Kanyári, Z., Bodnár, A., Kappelmayer, J. et al., Colorectal carcinoma rearranges cell surface protein topology and density in CD4+ T cells. Biochem. Biophys. Res. Commun. 2007, 361, 202-207.

[76] Wu, C. C., MacCoss, M. J., Howell, K. E., Yates, J. R., III, A method for the comprehensive proteomic analysis of membrane proteins. Nat. Biotechnol. 2003, 21, 532-538.

[77] Cao, R., He, Q., Zhou, J., He, Q. et al., High-throughput analysis of rat liver plasma membrane proteome by a nonelectrophoretic in-gel tryptic digestion coupled with mass spectrometry identification. J. Proteome Res. 2008, 7, 535545.

[78] Chick, J. M., Haynes, P. A., Molloy, M. P., Bjellqvist, B. et al., Characterization of the rat liver membrane proteome using peptide IPG IEF. J. Proteome Res. 2008, 7, 1036-1045. 